\title{
ABELIAN THEOREMS FOR WHITTAKER TRANSFORMS
}

\author{
RICHARD D. CARMICHAEL \\ Department of Mathematics and Computer Science \\ Wake Forest University \\ Winston-Salem, North Carolina 27109 \\ U. S. A. \\ and
}

\section{R.S. PATHAK}

Department of Mathematics

Banaras Hindu University

Varanasi 221005

India

(Received April 4, 1986 and In revised form October 29, 1986)

\begin{abstract}
Initial and final value Abelian theorems for the Whittaker transform of functions and of distributions are obtained. The Abelian theorems are obtained as the complex variable of the transform approaches 0 or $\infty$ in absolute value inside a wedge region in the right half plane.
\end{abstract}

KEY WORDS AND PHRASES. Abelian Theorems, Whittaker Transform of Functions and Distributions.

1980 AMS SUBJECT CLASSIFICATION CODE. 44A20, $46 \mathrm{~F} 12$.

1. INTRODUCTION.

Abelian theorems for the Whittaker transform of functions and of distributions are obtained as the complex variable of the transform approaches 0 or $\infty$ in absolute value inside a wedge region in the right half plane. The setting for these Abelian theorems is motivated by the initial and final value results of Doetsch [1, sections 33 and 34] for the Laplace transform of which the Whittaker transform is a generalization. Our results here generalize Abelian theorems previously obtained by Zemanian [2, sections 8.6 and 8.7], Akhaury [3-4], Moharir and Saxena [5], and Tiwari and Ko [6] in the generality of the transform variable being in a wedge and in the generality of the parameters.

2. THE WHITTAKER FUNCTION.

Let $k=k_{1}+i k_{2}$ and $m=m_{1}+i m_{2}$ be complex parameters which satisfy $\left(m_{1}-k_{1}+(1 / 2)\right)>0$. Let $s$ be a complex variable with $s \in \ell_{>}=\left\{s_{1}=s_{1}+i s_{2} \varepsilon \varnothing: s_{1}>0\right\}$. For $s \in l_{>}$the Whittaker function $W_{k, m}(s)$ (Erdélyi et al $[7,(2), p .264]$ ) is given by

$$
W_{k, m}(s)=\frac{e^{-s / 2} s^{m+(I / 2)}}{\Gamma(m-k+(1 / 2))} \int_{0}^{\infty} e^{-s u} u^{m-k-(1 / 2)}(1+u)^{m+k-(1 / 2)} d u .
$$

Let $\mathrm{p}$ be a positive real parameter. For $K \geq 0$ being a fixed real number put $P_{K}=\left\{s=s_{1}+i s_{2} \varepsilon \ell: s_{1}>0\right.$ and $\left.\left|s_{2}\right| \leq K s_{1}\right\} ; P_{K}$ is a wedge in the right half plane $C_{>}$. Using (2.1) with the gamma function taken to the left of the equality and estimate 
analysis on the exponentials and powers as in Carmichael and Hayashi [8, Lemma 2.2, p. 70] and Carmichael and Hayashi [8, proofs of Theorems 3.1 and 3.2], we have the following important estimate for this paper:

$$
\begin{aligned}
& \left|\mathrm{w}_{\mathrm{k}, \mathrm{m}}(\mathrm{ps} t)\right| \leq\left(1+\mathrm{K}^{2}\right)^{\left(2 \mathrm{~m}_{1}+1\right) / 4} \exp \left(\pi\left|\mathrm{m}_{2}\right| / 2\right) \frac{\Gamma\left(\mathrm{m}_{1}-\mathrm{k}_{1}+(1 / 2)\right)}{\Gamma(\mathrm{m}-\mathrm{k}+(1 / 2)) \mid} \mathrm{w}_{\mathrm{k}_{1}, \mathrm{~m}_{1}}\left(\mathrm{ps}_{1} t\right), \\
& s \varepsilon P_{K}, t>0 \text {. }
\end{aligned}
$$

3. THE WHITTAKER TRANSFORM FOR FUNCTIONS.

Let $\mathrm{k}, \mathrm{m}$, and $\mathrm{r}$ be complex parameters, and let $\mathrm{p}$ and $\mathrm{q}$ be real parameters. Let $\mathrm{s}$ be a complex variable. The function

$$
\mathrm{F}_{\mathrm{p}, \mathrm{q}, \mathrm{r}}^{\mathrm{k}, \mathrm{m}}(\mathrm{s})=\int_{0}^{\infty}(\mathrm{st})^{\mathrm{r}-(1 / 2)} \exp (-\mathrm{qst} / 2) \mathrm{w}_{\mathrm{k}, \mathrm{m}}(\mathrm{pst}) \mathrm{f}(\mathrm{t}) \mathrm{dt}
$$

is the Whittaker transform of the function $f(t)$ where $w_{k, m}$ (pst) is the Whittaker function. The general Whittaker transform defined in (3.1) was first considered by Srivastava [9] for certain values of the parameters and variable which depend upon the order of growth of $f(t)$.

In this section we prove initial and final value Abelian theorems for the 'Whittaker transform defined in (3.1). For this purpose we now place conditions on the parameters and variable noted above; these restrictions will hold throughout the remainder of this section. The complex parameters $k, m$, and $r$ satisfy $\operatorname{Re}(\mathrm{m}-\mathrm{k}+(1 / 2))>0$ and $\operatorname{Re}(\mathrm{r}) \geq \operatorname{Re}(\mathrm{m}) \geq 0$ with $\operatorname{Re}(\mathrm{r}) \geq(1 / 2)$. The real parameters $\mathrm{p}$ and $q$ are positive. The complex variable $s$ is in $\phi_{>}$, that is $\operatorname{Re}(s)>0$.

We now state and prove an initial value Abelian theorem.

THEOREM 3.1. Let $n=n_{1}+i n_{2}$ be complex with $\operatorname{Re}(n)=n_{1}>(-1)$. Let $f(t), 0 \leq t<\infty$, be a complex valued function such that there is a real number $c>0$ for which ( $\left.e^{-c t} f(t)\right)$ is absolutely integrable over $0 \leq t<\infty$ and such that $\left(f(t) / t^{n}\right)$ is bounded on $0<t \leq y$ for all $y>0$. Let the Whittaker transform $F_{p, q, r}^{k}(s)$ of $f^{t}(t)$ exist for $s \varepsilon k_{>}$. If there is a complex number $\alpha$ for which

$$
\lim _{t \rightarrow 0+} \frac{f(t)}{t^{n}}=\alpha
$$

then for each fixed $K \geq 0$

$$
\lim _{\substack{\mathrm{s} \mid \rightarrow \infty \\ \mathrm{s} \varepsilon \mathrm{P}_{\mathrm{K}}}} \frac{\mathrm{s}^{n+1} \mathrm{~F}_{\mathrm{p}, \mathrm{q}, \mathrm{m}}^{\mathrm{k}}(\mathrm{s})}{\Lambda(\eta, \mathrm{k}, \mathrm{m}, \mathrm{p}, \mathrm{q}, \mathrm{r})}=\alpha
$$

where

$$
\begin{aligned}
\Lambda(n, k, m, p, q, r)= & \frac{p^{m+(1 / 2)} \Gamma(n+m+r+1) \Gamma(n+r-m+1)}{((p+q) / 2)^{n+m+r+1} \Gamma(n+r-k+(3 / 2))} \\
& 2^{F}\left(n+m+r+1, m-k+(1 / 2) ; n+r-k+(3 / 2) ; \frac{q-p}{q+p}\right) .
\end{aligned}
$$

PROOF. Using Erdélyi et al [10, (16), p. 216] we have

$$
\int_{0}^{\infty} t^{n}(s t)^{r-(I / 2)} \exp (-q s t / 2) w_{k, m}(p s t) d t=s^{-n-1} \Lambda(n, k, m, p, q, r) .
$$


From (3.1) and (3.4) we obtain

$$
\begin{aligned}
& \left|s^{n+1} F_{p, q, r}^{k, m}(s)-\alpha \Lambda(n, k, m, p, q, r)\right|= \\
& =\left|s^{n+1} \int_{0}^{\infty}(s t)^{r-(1 / 2)} \exp (-q s t / 2) W_{k, m}(p s t)\left(f(t)-\alpha t^{n}\right) d t\right| \\
& \leq I_{1}+I_{2}
\end{aligned}
$$

where

$$
\begin{aligned}
& I_{1}=\left|s^{n+1} \int_{0}^{y}(s t)^{r-(1 / 2)} \exp (-q s t / 2) w_{k, m}(p s t)\left(f(t)-\alpha t^{n}\right) d t\right| \\
& I_{2}=\left|s^{n+1} \int_{y}^{\infty}(s t)^{r-(1 / 2)} \exp (-q s t / 2) w_{k, m}(p s t)\left(f(t)-\alpha t^{n}\right) d t\right|
\end{aligned}
$$

for $y>0$ arbitrary for the moment. For $s \in P_{K}$ we use the boundedness hypothesis on $\left(f(t) / t^{n}\right),(2.2)$, and estimates as in obtaining (2.2) to obtain

$$
\begin{aligned}
& I_{1}=\left|s^{n+1} \int_{0}^{y} t^{n}(s t)^{r-(1 / 2)} \exp (-q s t / 2) w_{k, m}(p s t)\left(\left(f(t) / t^{n}\right)-\alpha\right) d t\right| \\
& \leq\left(1+K^{2}\right)^{\left(n_{1}+m_{1}+r_{1}+1\right) / 2} \exp \left(\pi\left(\left|n_{2}\right|+\left|m_{2}\right|+\left|r_{2}\right|\right) / 2\right) \\
& \left(0<\sup _{t \leq y}\left|\left(f(t) / t^{n}\right)-\alpha\right|\right) s_{1}^{n_{1}+1} \frac{\Gamma\left(m_{1}-k_{1}+(1 / 2)\right)}{|\Gamma(m-k+(1 / 2))|} \\
& \int_{0}^{y} t^{n_{1}}\left(s_{1} t\right)^{r_{1}-(1 / 2)} \exp \left(-q s_{1} t / 2\right) w_{k_{1}, m_{1}}\left(p s_{1} t\right) d t
\end{aligned}
$$

The integrand in the integral of this last estimate is positive valued for $t \geq 0$ for the variable $s_{1}>0$ and the parameters $n_{1}, p, q, k_{1}, m_{1}$, and $r_{1}$. We thus replace the integral in the last estimate in (3.7) by an integral over $0<t<\infty$ and use (3.4) for the variable $s_{1}>0$ and the parameters $n_{1}, p, q, k_{1}, m_{1}$, and $r_{1}$; by so doing the estimate (3.7) can be continued as

$$
\begin{aligned}
I_{1} \leq\left(1+K^{2}\right)^{\left(n_{1}+m_{1}+r_{1}+1\right) / 2} \exp \left(\pi\left(\left|n_{2}\right|+\left|m_{2}\right|+\left|r_{2}\right|\right) / 2\right) \\
\quad \Lambda\left(n_{1}, k_{1}, m_{1}, p, q, r_{1}\right) \frac{\Gamma\left(m_{1}-k_{1}+(1 / 2)\right)}{|\Gamma(m-k+(1 / 2))|} \sup _{0<t \leq y}\left|\left(f(t) / t^{n}\right)-\alpha\right|
\end{aligned}
$$

and this bound is independent of $\mathrm{s} \varepsilon \mathrm{P}_{\mathrm{K}}$ and holds for each fixed $\mathrm{y}>0$. We now consider $I_{2}$ given in (3.6). By hypothesis there is a real number $c>0$ for which $\left(e^{-c t} f(t)\right)$ is absolutely integrable over $0 \leq t<\infty$. Putting $e^{-c t}$ into $I_{2}$ and using (2.2) and estimates for $s \in P_{K}$ we have 


$$
\begin{aligned}
& I_{2}=\left|s^{n+1} \int_{y}^{\infty}(s t)^{r-(1 / 2)} \exp (-(q s-2 c) t / 2) w_{k, m}(p s t)\left(e^{-c t}\left(f(t)-\alpha t^{n}\right)\right) d t\right| \\
& \leq\left(1+K^{2}\right)^{\left(n_{1}+m_{1}+r_{1}+1\right) / 2} \exp \left(\pi\left(\left|n_{2}\right|+\left|m_{2}\right|+\left|r_{2}\right|\right) / 2\right) s_{1}^{n_{1}+1} \frac{\Gamma\left(m_{1}-k_{1}+(1 / 2)\right)}{|\Gamma(m-k+(1 / 2))|} \\
& \int_{y}^{\infty}\left(s_{1} t\right)^{r_{1}-(1 / 2)} \exp \left(-\left(q s_{1}-2 c\right) t / 2\right) w_{k_{1}, m_{1}}\left(p s_{1} t\right)\left|e^{-c t}\left(f(t)-\alpha t^{n}\right)\right| d t .
\end{aligned}
$$

In this theorem we desire to prove (3.3) as $|s|+\infty$, $s \in P_{K}$. As $|s|+\infty$, s $\varepsilon P_{K}$, then necessarily $s_{1}=\operatorname{Re}(s) \rightarrow \infty$. Thus we now assume without loss of generality that $s_{1}=\operatorname{Re}(s)>(2 c / q)$ in the remainder of this proof for the fixed $c>0$ and $q>0$; and for $\operatorname{such} s_{1}=\operatorname{Re}(s)$ we know that

$$
\exp \left(-\left(q s_{1}-2 c\right) t / 2\right) \leq 1, t \geq 0 .
$$

As $t \rightarrow \infty$ then $\left(p_{1} t\right)+\infty$ here ince $p>0$ and $s_{1}>0$. From properties of $w_{k_{1}, m_{1}}\left(p s_{1} t\right)$ (Whittaker and Watson [11, Chapter 16]) Including the growth at $\infty$ (see also Tiwari and Ko $[6$, line $2 \downarrow, p .351]$ ) we have that

$\left(\left(p s_{1} t\right)^{r_{1}-(1 / 2)} w_{k_{1}, m_{1}}\left(p s_{1} t\right)\right)$ is positive, fintte valued, and continuous as a function of $t$ on $y \leq t<\infty$ and

$$
\lim _{t \rightarrow+\infty}\left(p s_{1} t\right)^{r_{1}-(1 / 2)} w_{k_{1}, m_{1}}\left(p s_{1} t\right)=0 ;
$$

thus $\left[\left(p_{1} t\right)^{r_{1}-(1 / 2)} w_{k_{1}, m_{1}}\left(p s_{1} t\right)\right]$ attains 1ts maximum at some point $t=t_{s_{1}}$ depending on $s_{1}$ such that $y \leq t_{s_{1}}<\infty$. Using this fact and $(3.10)$ we continue (3.9) as

$$
\begin{aligned}
& I_{2} \leq\left(1+K^{2}\right)^{\left(n_{1}+m_{1}+r_{1}+1\right) / 2} \exp \left(\pi\left(\left|n_{2}\right|+\left|m_{2}\right|+\left|r_{2}\right|\right) / 2\right) \\
& \quad \frac{\Gamma\left(m_{1}-k_{1}+(1 / 2)\right)}{|\Gamma(m-k+(1 / 2))|} s_{1}^{n_{1}+1} p^{-r_{1}+(1 / 2)}\left(p s_{1} t_{s_{1}}\right)^{r_{1}-(1 / 2)} w_{k_{1}, m_{1}}\left(p s_{1} t_{s_{1}}\right) \\
& \quad \int_{0}^{\infty}\left|e^{-c t}\left(f(t)-\alpha t^{n}\right)\right| d t \\
& \leq\left(1+K^{2}\right)^{\left(n_{1}+m_{1}+r_{1}+1\right) / 2} \exp \left(\pi\left(\left|n_{2}\right|+\left|m_{2}\right|+\left|r_{2}\right|\right) / 2\right)
\end{aligned}
$$




$$
\begin{aligned}
& \frac{\Gamma\left(m_{1}-k_{1}+(1 / 2)\right)}{|\Gamma(m-k+(1 / 2))|} p^{-r_{1}+(1 / 2)}(p y)^{-n_{1}-1}\left(p s_{1} t_{s_{1}}\right)^{n_{1}+r_{1}+(1 / 2)} \\
& w_{k_{1}, m_{1}}\left(p s_{1} t_{s_{1}}\right) \int_{0}^{\infty}\left|e^{-c t}\left(f(t)-\alpha t^{n}\right)\right| d t
\end{aligned}
$$

where the last inequality is obtained from the fact that $y \leq t_{s_{1}}<\infty$ and $n_{1}+1>0$. For $n_{1}=\operatorname{Re}(n)>(-1)$ we have $\left(t^{n} e^{-c t}\right)$ is absolutely integrable over $0<t<\infty$; this combined with the assumption in this theorem that $\left(e^{-c t} f(t)\right)$ is absolutely integrable over $0<t<\infty$ yield that the integral in the estimate (3.11) is finite. From the hypothesis (3.2) and the estimate (3.8), which is independent of $s \varepsilon P_{K}$, given $\epsilon / 2$ we can choose $y>0$ small enough so that $I_{1}<\epsilon / 2$. We now fix this $y>0$ and it depends only on $\epsilon>0$. As noted above, as $|s| \rightarrow+\infty$, s $\varepsilon P_{K}$, then necessarily $s_{1}=\operatorname{Re}(s) \rightarrow+\infty$, and as this happens $\left(p_{1} t_{s_{1}}\right) \rightarrow+\infty$ since $p>0$ is fixed and $y \leq t_{s_{1}}<\infty$. Using the growth property at $\infty$ of the Whittaker function $W_{k_{1}, m_{1}},(3.11)$ shows that $I_{2}$ can be made smaller than $\epsilon / 2$ if $|s|$ is chosen large enough, $s \in P_{K}$. Combining these facts with (3.5) yields the desired conclusion (3.3). The proof is complete.

As an example for which theorem 3.1 is applicable, let $f(t)=t /\left(1+t^{2}\right), n=1, c=1$, and $\alpha=1$. Another example is obtained if we take $f(t)=t^{2} /\left(1+t^{2}\right), n=1+1, c=1$, and $\alpha=0$.

We now obtain a final value Abelian theorem for the Whittaker transform of functions.

THEOREM 3.2. Let $n=n_{1}+i n_{2}$ with $n_{1}>(-1)$. Let $f(t), 0 \leq t<\infty$, be a complex valued function such that there is a real number $c>0$ for which $\left(e^{-c t} f(t)\right)$ is absolutely integrable over $0 \leq t<\infty$ and such that $\left(f(t) / t^{n}\right)$ is bounded on $y \leq t<\infty$ for all $y>0$. Let the Whittaker transform $F_{p, q, r}^{k, m}(s)$ of $f(t)$ exist for $s \varepsilon l_{>}$. If there is a complex number $\alpha$ for which

$$
\lim _{t \rightarrow+\infty} \frac{f(t)}{t^{n}}=\alpha
$$

then for each fixed $K \geq 0$

$$
\underset{s \in P_{K}}{\lim _{|s| \rightarrow 0}} \frac{s^{n+1} F_{p, q, r}^{k, m}(s)}{\Lambda(n, k, m, p, q, r)}=\alpha .
$$

PROOF. Using (3.4) and arguing as in (3.5) we have

$$
\left|s^{n+1} \underset{p, q, r}{k, m}(s)-\alpha \Lambda(\eta, k, m, p, q, r)\right| \leq I_{1}+I_{2}
$$

where $I_{1}$ and $I_{2}$ are defined in (3.6) for $y>0$ arbitrary. Let $K \geq 0$ be arbitrary but fixed and $s \in P_{K}$. Using the boundedness hypothesis on $\left(f(t) / t^{n}\right),(2.2)$, and analysis as in (3.7) and (3.8) we have 


$$
\begin{aligned}
I_{2} \leq\left(1+K^{2}\right)^{\left(n_{1}+m_{1}+r_{1}+1\right) / 2} \exp \left(\pi\left(\left|n_{2}\right|+\left|m_{2}\right|+\left|r_{2}\right|\right) / 2\right) \\
\Lambda\left(n_{1}, k_{1}, m_{1}, p, q, r_{1}\right) \frac{\Gamma\left(m_{1}-k_{1}+(1 / 2)\right)}{|\Gamma(m-k+(1 / 2))|}\left(\sup _{t \geq y}\left|\left(f(t) / t^{n}\right)-\alpha\right|\right)
\end{aligned}
$$

and the right side of (3.15) is independent of $s \in P_{K}$. We now consider $I_{1}$ in (3.6). For the real number $c>0$ in the hypothesis we argue as in (3.9) and obtain

$$
\begin{gathered}
I_{1} \leq\left(1+k^{2}\right)^{\left(n_{1}+m_{1}+r_{1}+1\right) / 2} \exp \left(\pi\left(\left|n_{2}\right|+\left|m_{2}\right|+\left|r_{2}\right|\right) / 2\right) \\
p^{-r_{1}+(1 / 2)} s_{1}^{n_{1}+1} \frac{\Gamma\left(m_{1}-k_{1}+(1 / 2)\right)}{|\Gamma(m-k+(1 / 2))|} \\
\int_{0}^{v}\left(p s_{1} t\right)^{r_{1}-(1 / 2)} \exp \left(-\left(q s_{1}-2 c\right) t / 2\right) w_{k_{1}, m_{1}}\left(p s_{1} t\right)\left|e^{-c t}\left(f(t)-\alpha t^{n}\right)\right| d t
\end{gathered}
$$

where we have put $p^{r_{1}-(1 / 2)}$ into the right side and hence have also put $p^{-r_{1}+(1 / 2)}$ there. The desired conclusion (3.13) in this theorem is to be obtained as $|s| \rightarrow 0$, $s \in P_{K}$; as $|s| \rightarrow 0$, $s \in P_{K}$, then necessarily $s_{I}=\operatorname{Re}(s) \rightarrow 0+$; we thus may assume without loss of generality here that $0<s_{1}<(1 / p)$ for the fixed parameter $p>0$. As $t \rightarrow 0+, 0<\mathrm{ps}_{1} t<t \rightarrow 0+$ for a.l $s_{1}<(1 / p)$; by the growth condition at 0 in Tiwari and Ko [6, line 4t, p. 351] or Whittaker and Watson [11, Chapter 16] we can choose constants $M$ and $T$ such that

$$
\begin{aligned}
\left|w_{k_{1}, m_{1}}\left(p s_{1} t\right)\right|=w_{k_{1}, m_{1}}\left(p s_{1} t\right) & \leq M\left(p s_{1} t\right)^{(1 / 2)-m_{1}} \\
0 & <p s_{1} t<t<T<y, s_{1}<(1 / p) ;
\end{aligned}
$$

here $M$ is independent of $s_{1}<(1 / p)$ and of $t<T$ and of $\mathrm{ps}_{1} t<t<T$ and $T$ is independent of $s_{1}<(1 / p)$. Returning to $(3.16)$ and using (3.17) and the fact that $\exp \left(-\mathrm{qs}_{1} t / 2\right) \leq 1, t>0$, we have

$$
\begin{aligned}
I_{1} \leq\left(1+K^{2}\right)^{\left(n_{1}+m_{1}+r_{1}+1\right) / 2} \exp \left(\pi\left(\left|n_{2}\right|+\left|m_{2}\right|+\left|r_{2}\right|\right) / 2\right) p^{-r_{1}+(1 / 2)} e^{c y} \\
s_{1}{ }^{\eta_{1}+1} \frac{\Gamma\left(m_{1}-k_{1}+(1 / 2)\right)}{|\Gamma(m-k+(1 / 2))|}\left(M\left(p s_{1} y\right)^{r_{1}-m_{1}} \int_{0}^{\infty}\left|e^{-c t}\left(f(t)-\alpha t^{n}\right)\right| d t\right. \\
\left.+\int_{T}^{y}\left(p s_{1} t\right)^{r_{1}-(1 / 2)} w_{k_{1}, m_{1}}\left(p s_{1} t\right)\left|e^{-c t}\left(f(t)-\alpha t^{n}\right)\right| d t\right) .
\end{aligned}
$$


In $(3.18)$, as in $(3.11)$, we have

$$
\int_{0}^{\infty}\left|e^{-c t}\left(f(t)-\alpha t^{n}\right)\right| d t<\infty
$$

$\left(e^{-c t} f(t)\right)$ is absolutely integrable over $0 \leq t<\infty$ by assumption and ( $e^{-c t} t^{n}$ ) is absolutely integrable over $0 \leq t<\infty$ since $c>0$ and $\operatorname{Re}(n)>(-1)$. Now $\left(\left(p_{1} t\right)^{r_{1}-(1 / 2)} w_{k_{1}, m_{1}}\left(p s_{1} t\right)\right)$ is a continuous function of $t$ on the closed bounded interval $\mathrm{T} \leq \mathrm{t} \leq \mathrm{y}$ for each fixed $\mathrm{s}_{1}, 0<\mathrm{s}_{1}<(1 / \mathrm{p})$; hence this product attains its maximum on $\mathrm{T} \leq \mathrm{t} \leq \mathrm{y}$ at a point $\mathrm{t}_{\mathrm{s}_{1}}$ depending on $\mathrm{s}_{1}<(1 / \mathrm{p})$. Continuing (3.18) we then have

$$
\begin{aligned}
I_{1} \leq\left(1+K^{2}\right)^{\left(\eta_{1}+m_{1}+r_{1}+1\right) / 2} \exp \left(\pi\left(\left|n_{2}\right|+\left|m_{2}\right|+\left|r_{2}\right|\right) / 2\right) p^{-r_{1}+(1 / 2)} & \frac{\left.p_{1}-k_{1}+(1 / 2)\right)}{|\Gamma(m-k+(1 / 2))|}\left(\cdot 1\left(p_{1} y\right)^{r_{1}-m_{1}}+\left(p_{1} t_{s_{1}}\right)^{r_{1}-(1 / 2)} w_{k_{1}, m_{1}}\left(p s_{1} t_{s_{1}}\right)\right) \\
& \int_{0}^{\infty y}\left|e^{-c t}\left(f(t)-\alpha t^{n}\right)\right| d t .
\end{aligned}
$$

The estimate (3.19) holds for all $s_{1}, 0<s_{1}<(I / p)$. The constants $c, K$, and $M$ are independent of $s_{1}, 0<s_{1}<(1 / p)$, as are all of the parameters $p, k=k_{1}+i k_{2}$, $m=m_{1}+i m_{2}, r=r_{1}+i r_{2}$ and $n=n_{1}+i n_{2}$ and the yet to be chosen constant $y>0$. Again as $|s| \rightarrow 0, s \varepsilon P_{K}$, then $s_{1}=\operatorname{Re}(s) \rightarrow 0+$. Recall that $T \leq t_{s_{1}} \leq y$ for $0<s_{1}<(1 / p)$ in (3.19) and $T$ is independent of $s_{1}, 0<s_{1}<(1 / p)$, but dependent on $\mathrm{y}>0$. (Recall (3.17).) As $\mathrm{s}_{1} \rightarrow 0+$ then $0<\mathrm{ps}_{1} \mathrm{t}_{\mathrm{s}_{1}} \leq \mathrm{ps}_{1} \mathrm{y} \rightarrow 0+$. Thus as $|s| \rightarrow 0$, $s \varepsilon P_{K}$, the growth condition Tiwari and Ko [6, line 4 $\left.\downarrow, p .351\right]$ and Whittaker and Watson [1l, Chapter 16] applied to $\mathrm{W}_{\mathrm{k}_{1}, \mathrm{~m}_{1}}\left(\mathrm{ps}_{1} \mathrm{t}_{\mathrm{s}_{1}}\right.$ ) yields constants $\mathrm{M}^{\prime}$ and $T^{\prime}$ which are independent of $s_{1}$ such that

$$
\begin{aligned}
& \left|\mathrm{w}_{\mathrm{k}_{1}, \mathrm{~m}_{1}}\left(\mathrm{ps}_{1} \mathrm{t}_{\mathrm{s}_{1}}\right)\right|=\mathrm{w}_{\mathrm{k}_{1}, \mathrm{~m}_{1}}\left(\mathrm{ps}_{1} \mathrm{t}_{\mathrm{s}_{1}}\right) \leq \mathrm{M}^{\prime}\left(\mathrm{ps}_{1} \mathrm{t}_{\mathrm{s}_{1}}\right)^{(1 / 2)-\mathrm{m}_{1},} \\
& 0<\mathrm{ps}_{1} \mathrm{t}_{1} \leq \mathrm{ps}_{1} \mathrm{y}<\mathrm{T}^{\prime},
\end{aligned}
$$

for $0<s_{1}<(1 / p)$. Using (3.20) in (3.19) and combining the resulting estimate on $I_{1}$ with the estimate $(3.15)$ on $I_{2}$, the proof can be completed similarly as in the proof of Theorem 3.1.

Examples of the applicability of Theorem 3.2 similar to those after the proof of Theorem 3.1 for Theorem 3.1 can be constructed by the reader. 
4. THE WHITTAKER TRANSFORM FOR DISTRIBUTIONS.

We construct a Whittaker transform for distributions as in Pathak [12]. We define the differential operators $D_{\alpha}, \alpha=0,1,2, \ldots$, as in Pathak $[12,(3)$, p. 4]; the seminorms $\gamma_{\alpha}, a=0,1,2, \ldots$, and the test functions $v_{a}(0, \infty)$ are given in Pathak $[12$, p. 6].

LEMMA 4.1. Let $s \neq 0$ and $\operatorname{Re}(s)>(a / p)$. Let $\operatorname{Re}(m) \geq 0$. We have $\left((s t)^{m-(1 / 2)} \exp (-p s t / 2) W_{k, m}(p s t)\right) \varepsilon V_{a}(0, \infty)$ as a function of $t \varepsilon(0, \infty)$.

PROOF. The proof is by Pathak [12, Lemma 1, p. 6]. Nothing different is introduced by having $\mathrm{p}>0$ in the exponential term and in $\mathrm{w}_{\mathrm{k}, \mathrm{m}}$ (pst) here.

Let $v_{a}^{\prime}(0, \infty)$ denote the space of generalized functions defined on $v_{a}(0, \infty)$ (Pathak $[12, p .6])$. Let $U \in v_{a}^{\prime}(0, \infty)$. Let $\sigma_{U}$ have the meaning given in Pathak $[12, p .7]$; that is $U \in v_{a}^{\prime}(0, \infty)$ for each a $>\sigma_{U}$ and $U \notin v_{a}^{\prime}(0, \infty)$ for any $a<\sigma_{U}$. Because of Lemma 4.1 we can form

$$
\mathrm{WT}_{\mathrm{p}}^{\mathrm{k}, \mathrm{m}}[\mathrm{U} ; \mathrm{s}]=\left\langle\mathrm{U}_{\mathrm{t}},(\mathrm{st})^{\mathrm{m}-(1 / 2)} \exp (-\mathrm{pst} / 2) \mathrm{w}_{\mathrm{k}, \mathrm{m}}(\text { pst })\right\rangle, \mathrm{s} \varepsilon \Omega_{\mathrm{U}},
$$

where $\Omega_{U}=\left\{\mathrm{s}: \mathrm{s} \neq 0, \operatorname{Re}(\mathrm{s})>\sigma_{U},-\pi<\operatorname{Arg}(\mathrm{s})<\pi\right\}$ and $\operatorname{Re}(\mathrm{m}) \geq 0$. We call $\mathrm{WT}_{\mathrm{p}}^{\mathrm{k}, \mathrm{m}}[\mathrm{U} ; \mathrm{s}]$ the distributional Whittaker transform of $U \varepsilon v_{a}^{\prime}(0, \infty)$; by Pathak [12, Theorem 1, p. 8] ${ }^{W T} T_{p}^{k, m}[U ; s]$ is an analytic function of $s$ in $\Omega_{U}$.

The set of seminorms $\left\{\gamma_{\alpha}\right\}, \alpha=0,1,2, \ldots$, which defines and generates the topology of $\mathrm{v}_{\mathrm{a}}(0, \infty)$ is a multinorm in the sense of Zemanian [13, p. 8] as noted in Pathak [12, p. 6]; here $\gamma_{0}$ is a norm. Thus the hypotheses of Zemanian [13, Theorem $1.8-1$, p. 18] are satisfied; by this result, given $U \in V_{a}^{\prime}(0, \infty)$ there exist a positive constant $C$ and $a$ nonnegative integer $N$, which depend only on $U$, such that

$$
|<U, \phi>| \leq C_{\alpha=0,1, \ldots, N}^{\max } \gamma_{\alpha}(\phi), \phi \varepsilon v_{\alpha}(0, \infty)
$$

We shall call the number $N$ here the order of the generalized function $U \varepsilon V_{a}^{\prime}(0, \infty)$.

We obtain Abelian theorems for the distributional Whittaker transform; in so doing we use the results of section 3. Thus in the remainder of this section we assume that the complex parameters $\mathrm{k}$ and $\mathrm{m}$ satisfy $\operatorname{Re}(\mathrm{m}-\mathrm{k}+(1 / 2))>0$. By comparing the forms (3.1) and (4.1) we see that the complex parameter $r$ and the real parameter $q$ of (3.1) and section 3 are now taken to be $r=m$ and $q=p$ in this section. We thus must make the restriction $\operatorname{Re}(\mathrm{m}) \geq(1 / 2)$ in the remainder of this section because $\operatorname{Re}(\mathbf{r})$ was necessarily so restricted in section 3 .

To prove our initial value theorem for $\mathrm{WT}_{\mathrm{p}}^{\mathrm{k}, \mathrm{m}}[\mathrm{U} ; \mathrm{s}]$ we need the following lemma.

LEMMA 4.2. Let $U \in V_{a}^{\prime}(0, \infty)$ for $a>\sigma_{U}$ and let the support of $U$ be in $T \leq t<\infty, T>0$. Let $s \varepsilon P_{K}$ with $(p \operatorname{Re}(s))>\max \left\{1, p \sigma_{U}, 4 a\right\}$. There are constants $\mathrm{B}>0$ and $\mathrm{y}_{0}>0$ which are independent of $\mathrm{s}$ such that

$$
\left|\mathrm{WT}_{\mathrm{p}}^{\mathrm{k}, \mathrm{m}_{\mathrm{m}}}[\mathrm{U} ; \mathrm{s}]\right| \leq B\left(1+\mathrm{s}_{1}\right)^{\mathrm{m}_{1}+\mathrm{k}_{1}+\mathrm{N}-(1 / 2)} \exp \left(-\mathrm{ps}_{1} \mathrm{y}_{0} / 4\right), \mathrm{s}_{1}=\operatorname{Re}(\mathrm{s})
$$


ABELIAN THEOREMS FOR WHITAKER TRANSFORMS

425

PROOF. The proof of this lemma is in the spirit of that of Zemanian [2, Lemma 1, p. 246]. Choose a function $\lambda(t) \varepsilon C^{\infty}$ such that for any nonnegative integer $\alpha$ we have

$$
\left|\frac{d^{\alpha}(\lambda(t))}{d t^{\alpha}}\right| \leq M_{\alpha},-\infty<t<\infty,
$$

where $M_{\alpha}$ is a constant which depends only on $\alpha$; $0 \leq \lambda(t) \leq 1$; $\lambda(t)=1$ for $T \leq t<\infty$; and the support of $\lambda(t)$, denoted $\operatorname{supp}(\lambda)$, is contained in $\mathrm{y}_{0} \leq t<\infty, 0<\mathrm{y}_{0}<\mathrm{T}$.

Then by the usual proof we have $\langle U, \phi\rangle=\langle U, \lambda \phi\rangle, \phi \varepsilon \mathrm{V}_{\mathrm{Q}}(0, \infty)$, since $\operatorname{supp}(\mathrm{U}) \subseteq[\mathrm{T}, \infty)$; and the value of $\langle U, \phi\rangle=\langle U, \lambda \phi\rangle$ is independent of the choice of $\lambda(t)$ satisfying the above properties. Let $\operatorname{Re}(s)>\sigma_{U}$. With $N$ being the order of $U \varepsilon v_{a}^{\prime}(0, \infty)$ we apply (4.2) and the definition Pathan [12, (12), p. 6] of the seminorms to obtain

$$
\begin{aligned}
& \left|\mathrm{WT}_{\mathrm{p}}^{\mathrm{k}, \mathrm{m}}[\mathrm{U} ; \mathrm{s}]\right|=\left|<\mathrm{U}_{t}, \lambda(\mathrm{t})(\mathrm{st})^{\mathrm{m}-(1 / 2)} \exp (-\mathrm{pst} / 2) \mathrm{W}_{\mathrm{k}, \mathrm{m}}(\mathrm{pst})>\right| \leq \\
& \leq C_{\alpha=0,1, \cdots, N}^{\max } 0<t<\infty \mid \mathrm{eup}^{\mathrm{at}} \mathrm{D}_{\alpha}\left(\lambda(\mathrm{t})(\mathrm{st})^{\mathrm{m}-(1 / 2)} \exp (-\mathrm{pst} / 2) \mathrm{W}_{\mathrm{k}, \mathrm{m}}\right. \text { (pst)) | } \\
& \text { for } \operatorname{Re}(s)>\sigma_{U} \text {. From Pathan }[12,(8), p .5] \text { we have } \\
& D_{\alpha}(f(t))=\sum_{\beta=0}^{\alpha} A_{\beta} t^{\beta} \quad f^{(\beta)}(t), \alpha=0,1,2, \ldots,
\end{aligned}
$$

where the $A_{\beta}$ are complex constants. Using (4.5), the Leibnitz rule, the boundedness of the derivatives of $\lambda(t)\left(\left|d^{\beta-\delta}(\lambda(t)) / d t^{\beta-\delta}\right| \leq M_{\beta, \delta}\right.$ in (4.6) below), and slater [14; $(2.4 .17)$, p. 25] we continue $(4.4)$ as

$$
\begin{aligned}
& \left|\mathrm{WTT}_{\mathrm{p}}^{\mathrm{k}, \mathrm{m}}[\mathrm{U} ; \mathrm{s}]\right| \leq
\end{aligned}
$$

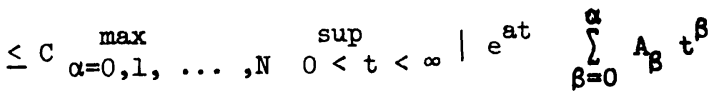

$$
\begin{aligned}
& \frac{d^{\beta}}{d t^{\beta}}\left[\lambda(t)(s t)^{m-(1 / 2)} \exp (-p s t / 2) w_{k, m}(p s t)\right) \mid
\end{aligned}
$$

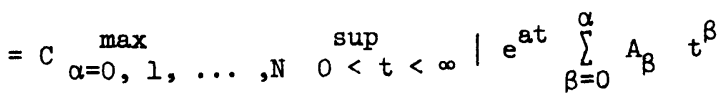

$$
\begin{aligned}
& \sum_{\delta=0}^{\beta} \frac{\beta !}{\delta !(\beta-\delta) !} \frac{d^{\beta-\delta}(\lambda(t))}{d t^{\beta-\delta}} \frac{d^{\delta}}{d t^{\delta}}\left[(s t)^{m-(1 / 2)} \exp (-p s t / 2) w_{k, m} \text { (pst) }\right) \mid
\end{aligned}
$$

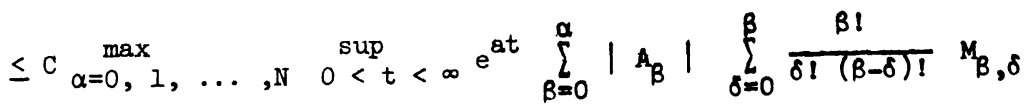

$$
\begin{aligned}
& \mid t^{\beta} p^{-m+(1 / 2)}(p s)^{\delta}(-1)^{\delta}(p s t)^{m-(1 / 2)-(\delta / 2)} \exp (-p s t / 2)
\end{aligned}
$$




$$
\begin{aligned}
& \mathrm{W}_{\mathrm{k}+(\delta / 2), \mathrm{m}-(\delta / 2)}(\mathrm{pst})
\end{aligned}
$$

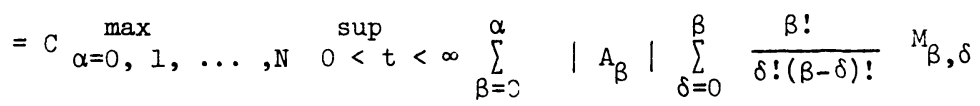

$$
\begin{aligned}
& \left|\mathrm{p}^{-\mathrm{m}+(1 / 2)+\delta-\beta}\right||\mathrm{s}|^{\delta-\beta} \mid \mathrm{e}^{a t}(\mathrm{pst})^{\mathrm{m}-(1 / 2)-(\delta / 2)+\beta} \exp (-\mathrm{pst} / 2) \\
& \mathrm{w}_{\mathrm{k}+(\delta / 2), \mathrm{m}-(\delta / 2)}(\mathrm{pst}) \mid
\end{aligned}
$$

Now recall $\mathrm{m}_{1}=\operatorname{Re}(\mathrm{m}) \geq(1 / 2),(\mathrm{p} \operatorname{Re}(\mathrm{s}))>\max \left\{1, \mathrm{p} \sigma_{\mathrm{U}}, 4 \mathrm{a}\right\}$, and $\mathrm{s} \varepsilon \mathrm{P}_{\mathrm{K}}$ in the hypothesis of this lemma; under these restrictions we use the growth properties of the Whittaker function (Whittaker and Watson [11, Chapter 16] and Tiwari and Ko [6, p. 351]) and analysis as in the proof of Pathak [12, Lemma 2, pp. 7-8] to obtain a constant $\mathrm{C}_{B}$ such that

$$
\begin{aligned}
\left|e^{a t}(\mathrm{pst})^{\mathrm{m}-(1 / 2)-(\delta / 2)+\beta} \exp (-\mathrm{pst} / 2) \mathrm{w}_{\mathrm{k}+(\delta / 2), \mathrm{m}-(\delta / 2)}(\mathrm{pst})\right| \leq \\
\leq \mathrm{C}_{B}\left(1+\mathrm{s}_{1}\right)^{\mathrm{m}_{1}+\mathrm{k}_{1}+\mathrm{N}-(1 / 2)} \exp \left(-\mathrm{ps} \mathrm{s}_{1} / 4\right)
\end{aligned}
$$

holds for $t>0$ and all relevant values of the parameters. Now in (4.6) we notice that $\left|\mathrm{p}^{-\mathrm{m}+(1 / 2)+\delta-\beta}\right|=\mathrm{p}^{-\mathrm{m}_{1}+(1 / 2)+\delta-\beta}$. Since $(\mathrm{p} \operatorname{Re}(\mathrm{s}))>\max \left\{1, \mathrm{po}_{\mathrm{U}}, 4 \mathrm{a}\right\}$ and $\mathrm{s} \varepsilon \mathrm{P}_{\mathrm{K}}$, the $\operatorname{term}|s|^{\delta-\beta}, \delta=0,1, \ldots, \beta, \beta=0,1, \ldots, \alpha$, in (4.6) is bounded by a constant which depends on $\delta$ and $\beta$. Now recall that $\operatorname{supp}(\lambda) \subseteq\left[\mathrm{y}_{0}, \infty\right)$ with $0<\mathrm{y}_{0}<\mathrm{T}$ so that the sup in $(4.4)$ and (4.6) is actually taken over $y_{0} \leq t<\infty$. Hence for the values of $t$ actually being considered in ( 4.4 ) and (4.6), the estimate in (4.7) holds for $y_{0} \leq t<\infty$ with the term $\exp \left(-\mathrm{ps}_{1} t / 4\right)$ replaced by $\exp \left(-\mathrm{ps}_{1} \mathrm{y}_{0} / 4\right)$. Combining these facts with (4.7) and (4.6) we obtain a constant $B$ which depends on the generalized function $U$ and on its order $N$ such that the desired conclusion (4.3) holds; the constants $B$ and $y_{0}$ are independent of s. The proof is complete.

We now prove an initial value Abelian theorem for the distributional Whittaker transform where the element $U \in V_{a}^{\prime}(0, \infty)$ is assumed to have support in $[0, \infty)$.

THEOREM 4.1. Let $U \in V_{\mathbf{a}}^{\prime}(0, \infty)$ for $a>\sigma_{U}$ such that over some right neighborhood $\left(0, t_{0}\right)$ of zero $U$ is a regular distribution corresponding to a complex valued function $f(t)$ such that there is a real number $c>0$ for which $\left(e^{-c t} f(t)\right)$ is absolutely integrable over $0 \leq t \leq t_{0}$. For $n=n_{1}+i n_{2}, n_{1}>-1$, let $\left(f(t) / t^{n}\right)$ be bounded on $0<t$ $\leq \mathrm{y}^{\prime}$ for all $\mathrm{y}^{\prime} \leq \mathrm{t}_{0^{\circ}}$ Let the Whittaker transform $\mathrm{F}_{\mathrm{p}, \mathrm{p}, \mathrm{m}}^{\mathrm{k}, \mathrm{m}}(\mathrm{s}), \mathrm{s} \varepsilon \phi_{>}$, exist for the function which is $f(t)$ on $0<t<y^{\prime}$ and which is zero on $y^{\prime} \leq t<\infty$ for all $y^{\prime} \leq t_{0}$. If there is a complex number $\alpha$ for which

$$
\lim _{t \rightarrow 0+} \frac{f(t)}{t^{n}}=\alpha
$$


then for each fixed $K \geq 0$

$$
\lim _{\mathrm{s} \mid \rightarrow \mathrm{P}_{K}} \frac{\mathrm{s}^{n+1} \mathrm{WT}^{\mathrm{k}, \mathrm{m}}[\mathrm{U} ; \mathrm{s}]}{\Lambda(\eta, \mathrm{k}, \mathrm{m}, \mathrm{p}, \mathrm{p}, \mathrm{m})}=\alpha .
$$

PROOF. We decompose $U$ into $U_{1}+U_{2}$ where $\operatorname{supp}\left(U_{1}\right) \subseteq[0, T]$ and $\operatorname{supp}\left(U_{2}\right) \subseteq[T, \infty)$, $0<\mathrm{T}<\mathrm{t}_{0}$. We now have

$$
\mathrm{WT}_{\mathrm{p}}^{\mathrm{k}, \mathrm{m}}[\mathrm{U} ; \mathrm{s}]=\mathrm{WT}_{\mathrm{p}}^{\mathrm{k}, \mathrm{m}}\left[\mathrm{U}_{1} ; \mathrm{s}\right]+\mathrm{WT}_{\mathrm{p}}^{\mathrm{k}, \mathrm{m}}\left[\mathrm{U}_{2} ; \mathrm{s}\right]
$$

Lemma 4.2 is applicable to $\mathrm{WT}_{\mathrm{p}}^{\mathrm{k}, \mathrm{m}}\left[\mathrm{U}_{2} ; \mathrm{s}\right]$ since $\operatorname{supp}\left(\mathrm{U}_{2}\right) \subseteq[\mathrm{T}, \infty), \mathrm{T}>0$. Recall that as $|s| \rightarrow \infty$, $s \in P_{K}$, then necessarily $s_{1}=\operatorname{Re}(s) \rightarrow \infty$. Thus by the conclusion (4.3) of Lemma 4.2 and the estimate

$$
\left|s^{n+1}\right| \leq\left(1+K^{2}\right)^{\left(n_{1}+1\right) / 2}\left(s_{1}\right)^{n_{1}+1} \exp \left(\pi\left|n_{2}\right| / 2\right) \text {, s } \varepsilon P_{K} \text {, }
$$

we have

$$
\lim _{s \in P_{K}} \frac{s^{n+1} \mathrm{WT}^{\mathrm{k}, \mathrm{m}}\left[\mathrm{U}_{2} ; s\right]}{\Lambda(n, k, m, p, p, m)}=0
$$

Extend the function $f(t)$ in the hypothesis, which is known on $0<t<t_{0}$, to $0<t<\infty$ by

$$
\bar{f}(t)=\left\{\begin{array}{c}
f(t), 0<t<T \\
0, T \leq t<\infty .
\end{array}\right.
$$

Then $\bar{f}(t)$ satisfies the hypotheses of Theorem 3.1 and $W T_{p}^{k, m}\left[U_{l} ; s\right]=W T_{p}^{k, m}[\bar{f}(t) ; s]$ with this latter Whittaker transform equaling the function Whittaker transform of $\bar{f}(t)$ defined in (3.1). Thus by Theorem 3.1

$$
\underset{s \in P_{K}}{\lim \mid \rightarrow \infty} \frac{s^{n+1} W_{T}^{k, m}\left[U_{1} ; s\right]}{\Lambda(n, k, m, p, p, m)}=\alpha .
$$

Combining (4.10), (4.11), and (4.12) we have the desired result (4.9). The proof is complete.

COROLLARY 4.1. Let $U \in V_{a}^{\prime}(0, \infty)$ for $a>\sigma_{U}$ such that over some right neighborhood $\left(0, t_{0}\right)$ of zero $U$ is a regular distribution corresponding to a function $f(t)$ which is Lebesgue integrable over $0 \leq t<\infty$. Let $n=n_{1}+i \eta_{2}, \eta_{1}>(-1)$. Let $\left(f(t) / t^{n}\right)$ be bounded on $0<t \leq y$ for all $y>0$ and let (4.8) be satisfied for some complex number $\alpha$. Then for each fixed $K \geq 0$ (4.9) holds. 
PROOF. Since $f(t)$ is Lebesque integrable over $0 \leq t<\infty$ then $\left(e^{-c t} f(t)\right)$ is absolutely integrable over $0 \leq t<\infty$ for any fixed $c>0$. The result thus follows immediately by Theorem 4.1.

We now proceed to obtain a final value Abelian theorem for the distributional Whittaker transform. First we need to make some comments concerning this transform. of a distribution of the form assumed in the final value theorem below. Let $U \varepsilon V_{a}^{\prime}(0, \infty), a>\sigma_{U}$, with $\operatorname{supp}(U) \subseteq\left[t_{0}, \infty\right), t_{0}>0$. Assume over some interval $\mathrm{y}<\mathrm{t}<\infty$ with $0<t_{\mathrm{C}}<\mathrm{y}$ that $\mathrm{U}$ is a regular distribution corresponding to a complex valued function $f(t)$. Then $U$ can be decomposed as $U=U_{1}+U_{2}$ with $\operatorname{supp}\left(U_{1}\right) \subseteq\left[t_{0}, T\right]$ and $\operatorname{supp}\left(\mathrm{U}_{2}\right) \subseteq[\mathrm{T}, \infty), \mathrm{T}>\mathrm{y}$. We then have

$$
\mathrm{WT}_{\mathrm{p}}^{\mathrm{k}, \mathrm{m}}[\mathrm{U} ; \mathrm{s}]=\mathrm{WT}_{\mathrm{p}}^{\mathrm{k}, \mathrm{m}}\left[\mathrm{U}_{1} ; \mathrm{s}\right]+\mathrm{WTT}_{\mathrm{p}}^{\mathrm{k}, \mathrm{m}}\left[\mathrm{U}_{2} ; \mathrm{s}\right]
$$

From the definition (4.1) $\mathrm{WT}_{\mathrm{p}}^{\mathrm{k}, \mathrm{m}}[\mathrm{U} ; \mathrm{s}]$ is defined for $\mathrm{s} \in \Omega_{\mathrm{U}}$ in which $\operatorname{Re}(\mathrm{s})>\sigma_{U}$. Because of the form of the $U \varepsilon V_{a}(0, \infty)$ assumed above and the assumptions on $f(t)$ in the final value Theorem 4.2 below, where we use the above decomposition, we can take $\sigma_{U}=0$ here, and (4.13) will be well defined in Theorem 4.2 for $s_{1}=\operatorname{Re}(s)>0$. Because of this we may let $|s| \rightarrow 0$, $s \in P_{K}$, in the final value theorem below as desired.

We now obtain a needed lemma for the final value result.

LEMMA 4.3. Let $U \in \mathcal{E}^{\prime}$ with $\operatorname{supp}(U) \subseteq\left[t_{0}, T\right], 0<t_{0}<T<\infty$. Let $n=n_{1}+i n_{2}$ with $n_{1}>-1$. For each fixed $k \geq 0$

$$
\underset{s \in P_{K}}{\lim \mid \rightarrow 0} s^{n+1} W^{k, m}[U ; s]=0 .
$$

PROOF. The distributional Whittaker transform $\mathrm{WT}_{\mathrm{p}}^{\mathrm{k}, \mathrm{m}}[\mathrm{U} ; \mathrm{s}]$ of $\mathrm{U} \varepsilon \mathcal{E}^{\prime}$ exists for $\operatorname{Re}(\mathrm{s})>0$. By Schwartz [15, Théorème, p. 91]

$$
U=\sum_{\alpha=0}^{N} \frac{d^{\alpha}\left(g_{\alpha}(t)\right)}{d t^{\alpha}}
$$

for some nonnegative integer $N$, the order of $U$, where the $g_{\alpha}(t), \alpha=0,1, \ldots, N$, are continuous functions with support in an arbitrary neighborhood $\left[t_{0}-\epsilon, T+\epsilon\right]$, $\boldsymbol{\epsilon}>0$, of $\left[t_{0}, T\right]$. Since $\boldsymbol{\epsilon}>0$ is arbitrary we assume here that $t_{0}-\epsilon>0$. Using distributional differentation and the calculation slater [14, (2.4.17), p. 25] we have

$\mathrm{WT}_{\mathrm{p}}^{\mathrm{k}, \mathrm{m}}[\mathrm{U} ; \mathrm{s}]=$

$$
=\sum_{\alpha=0}^{N}(-1)^{\alpha} \int_{t_{0}-\epsilon}^{T+\epsilon} g_{\alpha}(t) \frac{d^{\alpha}}{d t^{\alpha}}\left((s t)^{m-(1 / 2)} \exp (-p s t / 2) w_{k, m}(p s t)\right) d t
$$


$=\sum_{\alpha=0}^{N}(-1)^{\alpha} \int_{t_{0}-\epsilon}^{T+\epsilon} g_{\alpha}(t) p^{-m+(1 / 2)}(p s)^{\alpha}(-1)^{\alpha}(p s t)^{m-(1 / 2)-(\alpha / 2)}$

$$
\exp (-p s t / 2) w_{k+(\alpha / 2), m-(\alpha / 2)}(p s t) d t
$$

Now each $g_{\alpha}(t)$ is continuous on $t_{0}-\epsilon \leq t \leq T+\epsilon$; hence

$$
\left|g_{\alpha}(t)\right| \leq M_{\alpha}, t_{0}-\epsilon \leq t \leq T+\epsilon, \alpha=0,1, \ldots, N
$$

for constants $M_{\alpha}, \alpha=0,1, \ldots, N$. For each $\alpha=0,1, \ldots, N$ we have

$$
\left|s^{n+1+\alpha}\right| \leq\left(1+K^{2}\right)^{\left(n_{1}+1+\alpha\right) / 2} \exp \left(\pi\left|n_{2}\right| / 2\right) s_{1}{ }^{n_{1}+1+\alpha}, s \in P_{K}, n_{1}=\operatorname{Re}(n)>-1 \text {. }
$$

Using analysis like that in obtaining the estimate (4.7) in the proof of Lemma 4.2 we have

$$
\begin{aligned}
\mid(p s t)^{m-(1 / 2)-(\alpha / 2)} \exp (-p s t / 2) & w_{k+(\alpha / 2), m-(\alpha / 2)}(p s t) \mid \leq \\
& \leq c_{\alpha}\left(1+s_{1}\right)^{m_{1}+k_{1}-(1 / 2)} \exp \left(-p s_{1} t / 4\right)
\end{aligned}
$$

for constants $C_{\alpha}$ with $\alpha=0,1, \ldots, N$ and $s \in P_{K}$. Again recall that as $|s| \rightarrow 0$, s $\varepsilon P_{K}$, then necessarily $s_{1}=\operatorname{Re}(s) \rightarrow 0+$. Thus by combining (4.15), (4.16), $(4.17)$, and $(4.18)$ we obtain $(4.14)$ and the proof is complete.

We now obtain a final value Abelian theorem for the distributional Whittaker transform.

THEOREM 4.2. Let $U \varepsilon V_{a}^{\prime}(0, \infty)$, a $>\sigma_{U}$, with $\operatorname{supp}(U) \subseteq\left[t_{0}, \infty\right), t_{0}>0$. Over some interval $\mathrm{y}<\mathrm{t}<\infty, 0<\mathrm{t}_{0}<\mathrm{y}$, let $\mathrm{U}$ be a regular distribution corresponding to a complex valued function $f(t)$ for which there is a real number $c>0$ such that $\left(e^{-c t} f(t)\right)$ is absolutely integrable over $y \leq t<\infty$. For $n=\eta_{1}+i n_{2}, \eta_{1}>(-1)$, let $f(t) / t^{n}$ be bounded on $y^{\prime} \leq t<\infty$ for all $y^{\prime} \geq y$; and assume that $F_{p, p, m}^{k, m}(s), s \in l_{>}$, exists for the function which is $f(t)$ on $y^{\prime}<t<\infty$ and which is zero on $0 \leq t \leq y^{\prime}$ for all $y^{\prime} \geq y$. If there is a complex number $\alpha$ for which

$$
\lim _{t \rightarrow+\infty} \frac{f(t)}{t^{n}}=\alpha
$$

then for each fixed $K \geq 0$

$$
\underset{s \in P_{K}}{\lim \mid \rightarrow 0} \frac{s^{n+1} W T_{p}^{k, m}[U ; s]}{\Lambda(n, k, m, p, p, m)}=\alpha
$$


PROOF. Decompose $U$ into $U=U_{1}+U_{2}$ with $\operatorname{supp}\left(U_{1}\right) \subseteq\left[t_{0}, T\right]$ and $\operatorname{supp}\left(U_{2}\right) \subseteq[t, \infty), T>y$, as in the paragraph above which contains equation (4.13); and by the discussion in that paragraph, (4.13) holds for $s_{1}=\operatorname{Re}(s)>0$. Let the function $f(t)$ in the hypothesis, which is known on $y<t<\infty$, be extended to $0 \leq t<\infty$ by

$$
\bar{f}(t)= \begin{cases}0, & 0 \leq t \leq T, \\ f(t), & T<t<\infty .\end{cases}
$$

Then $\bar{f}(t)$ satisfies the hypotheses of Theorem 3.2. Now $W^{\mathrm{k}}{ }_{\mathrm{p}}^{\mathrm{m}}\left[\mathrm{U}_{2} ; \mathrm{s}\right]=\mathrm{WT}_{\mathrm{p}}^{\mathrm{k}, \mathrm{m}}[\overline{\mathrm{f}}(\mathrm{t}) ; \mathrm{s}]$ with this latter Whittaker transform equaling the function Whittaker transform of $\bar{f}(t)$ defined in (3.1). Thus the conclusion (4.20) follows from (4.13), Lemma 4.3, and Theorem 3.2. The proof is complete.

In future work we hope to extend the Abelian theorems of Joshi and Saxena [16] and Malgonde and Saxena [17] for the H-transform to the general setting that the complex variable of the transform approaches 0 or $\infty$ inside a wedge region in the right half plane and for more general parameters, To do so we will need to use the properties of the H-function as in Srivastava et al [18]. Analysis which is associated with that in this paper and with the corresponding H-transform problem is contained in Sinha [19] and Joshi and Saxena [20]. The Meijer transform is studied in Pathak [21] which we note here because of the similarity of the Meijer and Whittaker transforms. 5. ACKNOWLEDGEMENTS.

In a letter to one of us (R.D.C.) Professor H. M. Srivastava has made some comments concerning certain analysis contained in this paper. We thank Professor Srivastava for this contribution to our paper.

One of us (R.D.C.) expresses his sincere appreciation to the Department of Mathematical Sciences of New Mexico State University for the opportunity of serving as Visiting Professor during 1984-1985 when the research on this paper began.

The research of Richard D. Carmichael in this paper is based upon work supported by the National Science Foundation under Grant No. DMS-8418435.

Some of the research of R. S. Pathak in this paper is based upon work which was partially supported by a grant from the William C. Archie Fund for Faculty Excellence of Wake Forest University. This author expresses his appreciation for this support while visiting Wake Forest University.

\section{REFERENCES}

1. DOETSCH, G. Introduction to the Theory and Application of the Laplace Transformation, Springer-Verlag, New York, 1974.

2. ZEMANIAN, A. H. Distribution Theory and Transform Analysis, McGraw-Hill, New York, 1965.

3. AKHAURY, S. K. Abelian Theorems for a Distributional Generalized Laplace Transform, Ranchi Univ. Math. J. 3 (1972), 82-89.

4. AKHAURY, S. K. Abelian Theorems for a Distributional Generalized Laplace Transform II, Ranchi Univ. Math. J. 12 (1981), 42-50.

5. MOHARIR, S. K. and R. K. SAXENA. Abelian Theorems for the Generalized Whittaker Transform, J. Shivaji Univ. 18 (1979), 7-11. 
6. TIWARI, A. K. and A. KO. Certain Properties of a Distributional Generalized Whittaker Transform, Indian J. Pure Appl. Math. 13 (1982), 348-361.

7. ERDÉLYI, A., W. MAGNUS, F. OBERHETTINGER, and F. G. TRICOMI. Higher Transcendental Functions, Vol. I, McGraw-Hill, New York, 1953.

8. CARMICHAEL, Richard D. and Elmer K. HAYASHI. Abelian Theorems for the Stieltjes Transform of Functions, II, Internat. J. Math. Math. Sci. 4 (1981), 67-88.

9. SRIVASTAVA, H. M. Certain Properties of a Generalized Whittaker Transform, Mathematica 10 (1968), 385-390.

10. ERDÉLYI, A., W. MAGNUS, F. OBERHETTINGER, and F. G. TRICOMI. Tables of Integral Transforms, Vol. I, McGraw-Hill, New York, 1954.

11. WHITTAKER, E. T. and G. N. WATSON. A Course of Modern Analysis, Fourth Ed., Cambridge University Press, London, 1927.

12. PATHAK, R. S. Transformée de Varma des Fonctions Généralisées, Bull. Sci. Math. 99 (1975), 3-16.

13. ZEMANIAN, A. H. Generalized Integral Transformations, Interscience Publishers, New York, 1968.

14. SLATER, L. J. Confluent Hypergeometric Functions, Cambridge University Press, London, 1960.

15. SCHWARTZ, L. Théorie des Distributions, Hermann, Paris, 1966.

16. JOSHI, V. G. and R. K. SAXENA. Abelian Theorems for Distributional H-Transform, Math. Ann. 256 (1981), 311-321.

17. MALGONDE, S. P. and R. K. SAXENA. Some Abelian Theorems for the Distributional H-Transformation, Indian J. Pure Appl. Math. 15 (1984), 365-370.

18. SRIVASTAVA, H. M., K. C. GUPTA, and S. P. GOYAL. The H-Functions of One and Two Variables with Applications, South Asian Publishers, New Delhi and Madras, 1982 .

19. SINHA, S. K. A Complex Inversion Formula and a Tauberian Theorem for the Generalized Whittaker Transform, Jñ̄näbha 11 (1981), 31-39.

20. JOSHI, V. G. and R. K. SAXENA. Complex Inversion and Uniqueness Theorem for the Generalized H-Transform, Indian J. Pure Appl. Math. 14 (1983), 322-329.

21. PATHAK, R. S. On the Meijer Transform of Generalized Functions, Pacific J. Math. 80 (1979), 523-536. 


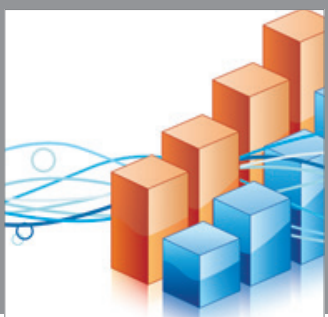

Advances in

Operations Research

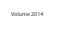

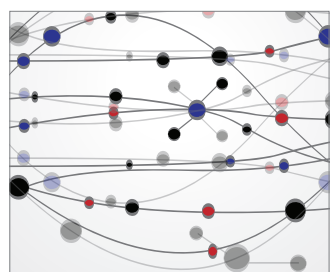

\section{The Scientific} World Journal
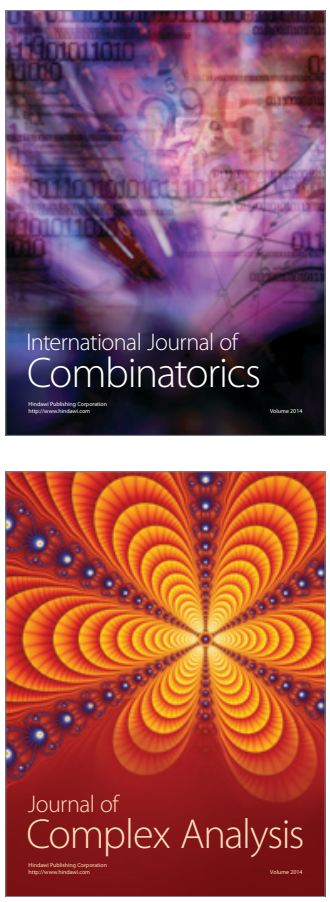

International Journal of

Mathematics and

Mathematical

Sciences
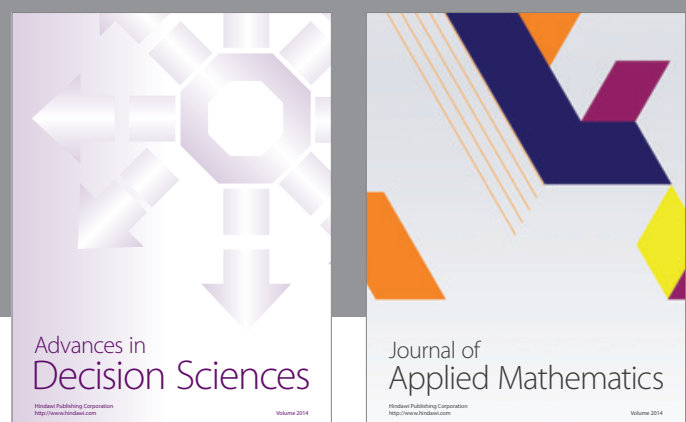

Journal of

Applied Mathematics
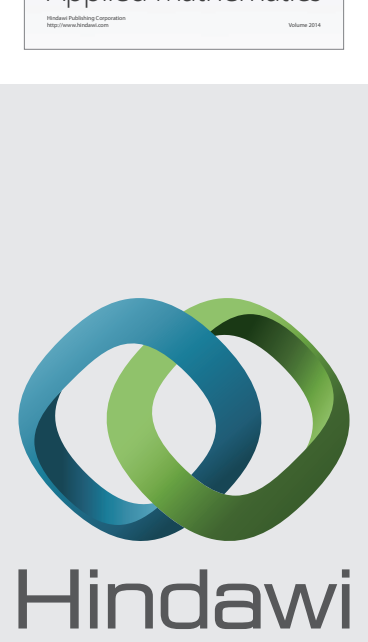

Submit your manuscripts at http://www.hindawi.com
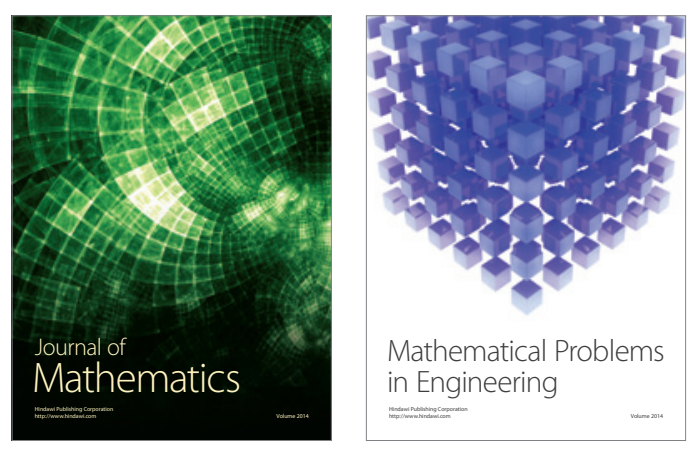

Mathematical Problems in Engineering
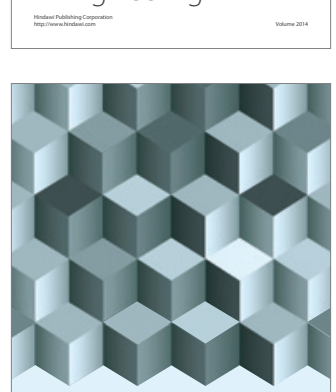

Journal of

Function Spaces
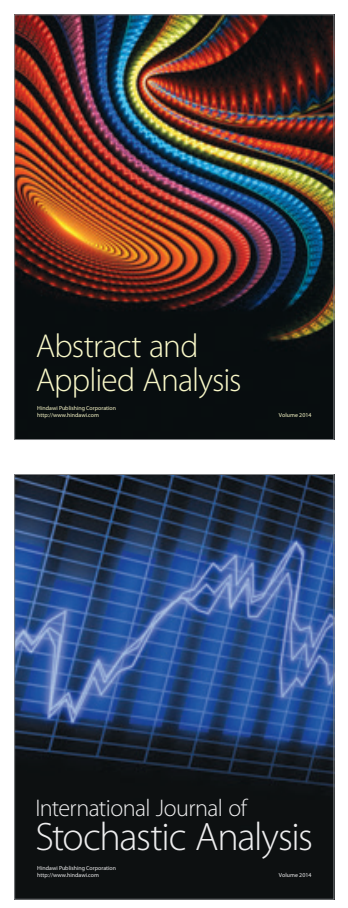

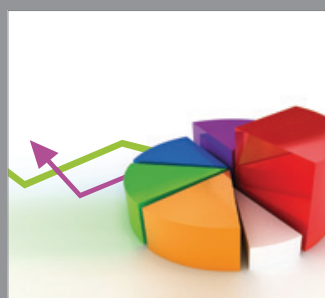

ournal of

Probability and Statistics

Promensencen
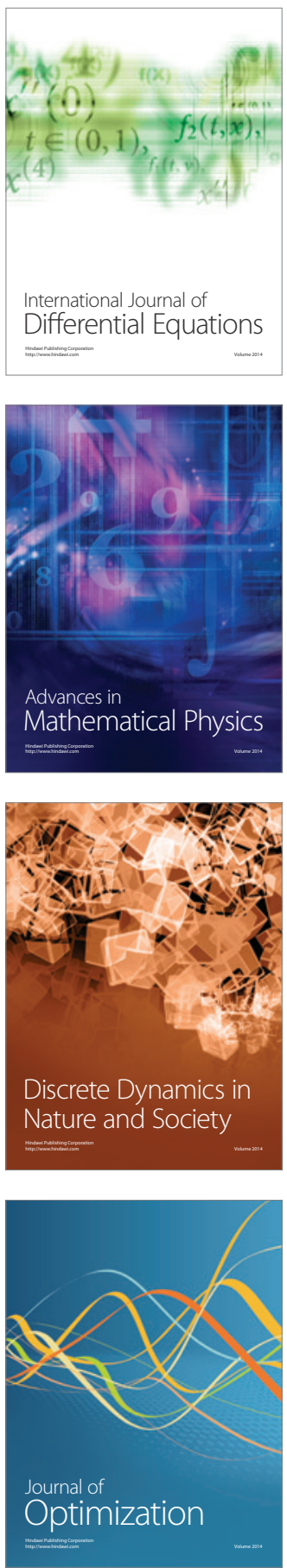